Check for updates

Cite this: RSC Adv., 2017, 7, 54835

\title{
Blue-white screening as a new readout for deoxyribozyme activity in bacterial cells $\uparrow$
}

\begin{abstract}
S. Sadeghi, N. Ahmadi, A. Esmaeili (iD * and F. Javadi-Zarnaghi (D)*
The 10-23 deoxyribozyme is considered as sequence-specific "molecular scissors" for RNA molecules. Extensive investigations have been reported for this deoxyribozyme in vitro and in eukaryotic host cells. However, few investigations are reported in the literature on the activity of this deoxyribozyme inside bacterial cells. The available reports focus on the cleavage of target mRNAs that encode for proteins which are responsible for the viability of bacterial colonies. Hence, the growth of bacterial cells was blocked and the main readouts in such studies were colony counts or optical density at $600 \mathrm{~nm}$. In the current study, blue-white screening was utilized as a novel readout for analysis of the activity of the 1023 deoxyribozymes in viable bacterial cells. Two deoxyribozymes were designed to target the $\alpha$-peptide fragment from $\beta$-galactosidase (lacZ) mRNA at two different positions, i.e. $5^{\prime}$ untranslated region and translated region. Control experiments were performed utilizing DNA oligos that lacked the catalytic core. The 3'-3 inverted thymidine modified deoxyribozymes were compared with unmodified ones to analyze the effect of such modification in prokaryotic cells. The activity of the designed deoxyribozymes caused a significant retardation in the formation of the blue-color in colonies with deoxyribozymes. Miller assay confirmed the blue-white screening results. This report showed a proof of concept for application of blue-white screening as a readout system for the activity of the 10-23 deoxyribozyme that is a model RNA-cleaving deoxyribozyme. The result of this report can promote future investigations on the activity of deoxyribozymes in prokaryotic cells.
\end{abstract}

Received 31st August 2017 Accepted 23rd November 2017

DOI: 10.1039/c7ra09679h

rsc.li/rsc-advances

\section{Introduction}

Protein enzymes were known as the sole biological catalysts until the 1980s. During the 1980s two natural RNA catalysts (ribozymes) were discovered for which a Nobel prize was shared between two scientists, Sidney Altman and Thomas Cech in 1989. ${ }^{1}$ A few years later, reports on oligodeoxyribonucleotidemediated catalysis demonstrated catalytic ability of DNA molecules. ${ }^{2}$ Since then, a growing number of deoxyribozymes (DNAzymes, DNA catalysts, Dzs) have been selected in vitro. ${ }^{3}$ Early evolved deoxyribozymes were RNA-cleaving ones. ${ }^{2 b, 4}$ The catalytic cores of these deoxyribozymes, like many others, were flanked by two binding arms that complementarily bind specific RNA substrates.

RNA-cleaving deoxyribozymes can be considered as sequence-specific "molecular scissors" for RNA molecules. The 10-23 deoxyribozyme which consists of a 15 nucleotide long catalytic core is a well-known example of an RNA-cleaving deoxyribozyme. It was shown that this deoxyribozyme can cleave biologically relevant RNA molecules in vitro in a site

Cell, Molecular Biology and Biochemistry Division, Department of Biology, Faculty of Sciences, University of Isfahan, Isfahan, Iran. E-mail: aesmaeili@sci.ui.ac.ir; fa.javadi@sci.ui.ac.ir

$\dagger$ Electronic supplementary information (ESI) available. See DOI: 10.1039/c7ra09679h specific manner. ${ }^{5}$ The activity of the 10-23 deoxyribozyme was also monitored in vivo. ${ }^{6}$ The enzyme was shown to be capable to modulate gene expression at post-transcriptional level. ${ }^{7}$

The in vivo targets of 10-23 deoxyribozyme were mostly viral RNA $^{8}$ or mRNA of eukaryotic cells. ${ }^{9}$ Many genes from HIV-1, ${ }^{10}$ hepatitis $\mathrm{B}$ and $\mathrm{C},{ }^{11}$ influenza $\mathrm{A}$ and $\mathrm{B},{ }^{\mathbf{1 2}}$ human papilloma virus, ${ }^{13}$ severe acute respiratory syndrome coronavirus (SARS) ${ }^{\mathbf{1 4}}$ and Epstein-Barr virus have been the targets for 10-23 deoxyribozyme RNA cleavage. ${ }^{15}$ Handful of genes responsible for cardiovascular, inflammatory or central nervous system diseases were down-regulated with 10-23 deoxyribozyme. ${ }^{6}$ Many cancer related genes such as multidrug resistance gene (MDR) have been targeted with this enzyme. ${ }^{16}$ Currently, the $10-$ 23 deoxyribozyme is a candidate therapeutic for several human diseases such as allergic bronchial asthma with $\mathrm{T}_{\mathrm{H}} 2$ molecular signature. ${ }^{17}$ The GATA-3 targeting $10-23$ deoxyribozyme is now under clinical trial investigations by sterna biologicals and phase IIa for early-phase and late phase asthmatic responses after allergen provocation has been completed. ${ }^{18}$

Despite advances in application of 10-23 deoxyribozyme in eukaryotic and mammalian cells, limited numbers of reports are available on investigation of the 10-23 deoxyribozyme activity inside the bacterial cells. ${ }^{6}$ A major challenge in application of deoxyribozymes in bacterial cells is dilution of the transfected oligonucleotide inside the fast dividing cells. Thus, all present 
reports used the 10-23 deoxyribozyme in an antibiotic-like manner. Such reports focus on targeting mRNAs of genes that were essential for viability and proliferation of the bacterial cells. The mRNAs for genes that are responsible for antibiotic resistance such as, mecR1 (ref. 23b) and bla1 (ref. 23a) related to the $\beta$-lactamase pathway are the most targets for application of $10-$ 23 deoxyribozyme in bacterial cells. Besides, Tan et al. reported cleavage of ftsZ mRNA by 10-23 deoxyribozyme. ${ }^{24}$ FtsZ gene is responsible for binary division of $E$. coli cells and its downregulation prevents bacterial colony formation. Therefore, in all the reported cases for prokaryotic application of 10-23 deoxyribozyme, inhibition of cell growth was the main result of the activity of the deoxyribozyme. Counting colonies on agar plate or analyzing $\mathrm{OD}_{600}$ were the main readout systems for such studies.

Due to the aforementioned technical issues, the current applications of deoxyribozymes in prokaryotic cells are limited to 10-23 and cleavage of vital mRNAs. Although inhibition of bacterial growth has advantages and permits application for controlling bacterial populations, indeed it does not grant investigation of the activity of the deoxyribozyme inside of the prokaryotic cells. Cells where the deoxyribozyme are active die due to the blockade of the target genes and thus no means are available to understand the mechanism of action of the deoxyribozyme in viable bacterial cells. Fundamentally, it would be helpful to have a new readout system for investigation of the activity of various deoxyribozymes inside vital prokaryotic cells. In addition, with deeper understanding on the kinetics of deoxyribozymes inside prokaryotic cells, the scope is capable of broadening e.g. to utilize the prokaryotic-active deoxyribozymes as biological elements of biosensors.

The lactose (lac) operon consists of three genes for production of $\beta$-galactosidase ( $\operatorname{Lac} Z$ ), permease (Lac $Y$ ) and transacetylase $(\operatorname{Lac} A)$. The lac operon is widely used as a reporter of gene insertion in cloning at molecular biology labs by simple and visual blue-white screening. The enzyme $\beta$-galactosidase is a tetramer with identical four polypeptides and is responsible for formation of a blue color upon presence of a chromogenic substrate like 5-bromo-4-chloro-3-indolyl- $\beta$-D-galactopyranoside (X-gal). Deletion of a few amino acids from amino-terminus of monomers of the enzyme, causes the formation of a dimer which may also further breaks down to monomers. Administration of the "missing" amino acids in an oligopeptide, also called $\alpha$-peptide results in re-structuring of the enzyme in active tetramer form. Molecular biologists hijacked this mechanism to analyze cloning efficiency. ${ }^{26}$ To this endeavor, the host must be an $E$. coli strain with missing amino acids from amino-terminus of $\beta$-galactosidase. Upon transformation, the $\alpha$-peptide gets provided into the cells. For a standard blue-white screening, the gene of interest is inserted in a multiple cloning site which is located inside the coding sequence of the $\alpha$-peptide. Thus, upon gene insertion, the $\alpha$-peptide is disrupted and $\alpha$-complementation may not occur. In contrast, vectors with complete and active $\alpha$-peptide will perform $\alpha$-complementation. As a result, the colonies with vector that contains the gene of interest do not bear active tetramer $\beta$-galactosidase and cannot produce a blue color from X-gal, while colonies with vectors that lack the gene of interest will form a blue color. Accordingly, the color of the colonies will be a marker for of the activity of the $\beta$-galactosidase and consequently presence of the gene of interest.

This report provides a novel application for the well-known method of blue-white screening. The presented method doesn't utilize the screening as a readout of gene insertion but rather it pursues the catalytic activity of the 10-23 deoxyribozyme. Here, the fully active $\alpha$-peptides were transformed into bacterial hosts. The 10-23 deoxyribozymes targeted the mRNA of the $\alpha$-peptides. The post-transcriptional cleavage of the mRNA of the $\alpha$-peptide was expected to diminish $\alpha$ complementation and formation of the blue color. Hence, the proposed method is able to report the activity of the $\beta$-galactosidase in viable cells. Proof of principle results were shown for the activity of the 10-23 deoxyribozyme for site specific cleavage at $5^{\prime}$-UTR and translated regions.

In most of the few available reports on prokaryotic cells, the oligodeoxyribonucleotide sequences of 10-23 deoxyribozyme were transfected to the bacterial cell as naked single stranded DNA. As an example, in a case report, oligonucleotides bearing the sequence of 10-23 deoxyribozyme as monomers and dimers designed against $\beta$-lactamase gene and was investigated in TEM-1 producing E. coli. $^{19}$

Naked unmodified oligonucleotides are prone to degradation by nucleases. Various types of DNA modifications are available to block nuclease degradation of oligonucleotides in vivo. Addition of a $3^{\prime}$ inverted nucleotide at the $3^{\prime}$ end of the oligo, ${ }^{20}$ phosphorothioation of the oligo backbone, ${ }^{21}$ modification at $2^{\prime}$ position of the ribose sugar such as $2^{\prime}$-O-methyl modification $^{22}$ and application of locked nucleic acid (LNA) ${ }^{13}$ on deoxyribozymes' binding arms are common examples. Among such a wide spectrum of DNA modifications, only phosphorothioation of the 10-23 deoxyribozyme was investigated in prokaryotic cells. Phosphorothioated DNA oligos were applied to cleave $\beta$-lactamase in oxacillin and methicillin resistant $S$. aureus, WHO-2 strains. ${ }^{23}$ In another strategy, vectors have been designed to transform the bacterial cells with deoxyribozymes 10-23. pssXGa vector ${ }^{24}$ and pBlue-script-II KS (+) phagemid $^{25}$ were designed for $E$. coli. In such strategies, the deoxyribozymes are induced to be synthesized inside bacteria and thus there is less concern for nuclease degradation. In this report, modified and unmodified DNA oligos with $3^{\prime}-3^{\prime}$ inverted thymidine were compared for blockade of the activity of the $\beta$ galactosidase. In addition, the effect of the deoxyribozymes was dissected from antisense effect, by comparing the results of full deoxyribozymes and the ones without catalytic core.

\section{Results}

\section{Design of deoxyribozymes}

The promoter of the lacZ- $\alpha$ peptide fragment was located in the reverse orientation from nucleotides 210-239 of the pGEM-T vector. Thus, the transcript template starts from position 209 in the reverse complement form down to position 2832, encompassing multiple cloning site and SP6 and m13 primer binding sites. The complete pGEM-T vector sequence with assigned fragments is depicted in ESI Appendix SI. $\dagger$ The mRNA transcript of the $\alpha$-peptide fragment with pGEM-T vector was 
$378 \mathrm{nt}$ long. The sequence of the transcript is provided in ESI Appendix SII. $\dagger$ The transcript coded for a fragment of lacZ $\alpha$ peptide from amino acid 67-115. The sequence of the $\alpha$-peptide fragment from pGEM-T is presented in ESI Appendix SIII. $\dagger$ The start codon on the transcribed mRNA was at position 44-47.

The predicted secondary structure of the transcript was taken as a guide to choose the best cleavage sites. To support effective approach of the deoxyribozymes's binding arms to the target mRNA, positions at which no extensive stems were present have been selected as target sites. Extended binding arms would thermodynamically inhibit binding of the deoxyribozymes to the target. To address the effect of the position of the cleavage site, two target sites were chosen; one in the $5^{\prime}$-untranslated region $\left(5^{\prime}\right.$ UTR), upstream of the first AUG and the second in the translated region. The 10-23 deoxyribozyme is known to be specific for cleavage at purine-pyrimidine junctions. The $A_{39}-C_{40}$ junction (inside $5^{\prime}$ UTR) and $A_{106}-C_{107}$ junction (inside translated region) were selected for cleavage by the deoxyribozymes. The deoxyribozymes were applied in two formats; with $3^{\prime}-\mathrm{OH}$ and with $3^{\prime}-3^{\prime}$ inverted thymidine. The latter was used to investigate protection potentials of such modification in prokaryotic host cells. The effect of three control DNAs was analyzed as well; (1) to dissect the catalytic activity of the designed deoxyribozymes from antisense effect, DNA oligos without catalytic cores (WOCC) were analyzed in parallel. WOCC had three thymidines instead of the catalytic core. (2) Additionally, a DNA oligonucleotide that do not target lacZ mRNA was co-transformed with PGEM-T vector as non-target DNA control (NTC). (3) Besides, in a negative control experiment, pGEM-T was transformed solely to $E$. coli competent cells. Fig. 1 and Table 2 depict the schematic structure of the target mRNA and sequences of the designed deoxyribozymes respectively.

\section{Magnesium chloride optimization}

Kinetic studies on 10-23 deoxyribozyme, similar to many others, confirmed strong dependence of the enzyme on bivalent metal ions e.g. $\mathrm{Mg}^{2+} \cdot{ }^{4}$ The activity of this deoxyribozyme is increased upon increment in $\mathrm{Mg}^{2+}$ concentration in a hyperbolic manner. ${ }^{4}$ However, cellular concentration of $\mathrm{Mg}^{2+}$ is limited to submillimolar concentrations. Thereupon, the activity of deoxyribozymes gets hampered. Specific pumps, channels etc. guarantee a balanced concentration of $\mathrm{Mg}^{2+}$ inside cells, and the $\mathrm{Mg}^{2+}$ concentration of the culture media do not directly represent its cellular concentration. However, to assure that adequate amount of $\mathrm{Mg}^{2+}$ is available, the bacterial growth at various concentrations of $\mathrm{Mg}^{2+}$ was analyzed. The effect of $\mathrm{Mg}^{2+}$ on the cell viability of $E$. coli colonies were investigated on agar plates. The highest concentration of $\mathrm{Mg}^{2+}$ with no inhibition of cell growth was considered to be optimal. To this end, we investigated at which concentration of $\mathrm{Mg}^{2+}$ the cell viabilities start to drop. Our result showed that $\mathrm{Mg}^{2+}$ concentration above $40 \mathrm{mM}$ reduces colony formation and thus, we decided to perform experiments with $35 \mathrm{mM} \mathrm{Mg}^{2+}$ present in the culture media (Fig. 2).

\section{Blue-white screening}

Upon transformation and subsequent incubation on ampicillin containing agar medium, white colonies get formed in a time
A
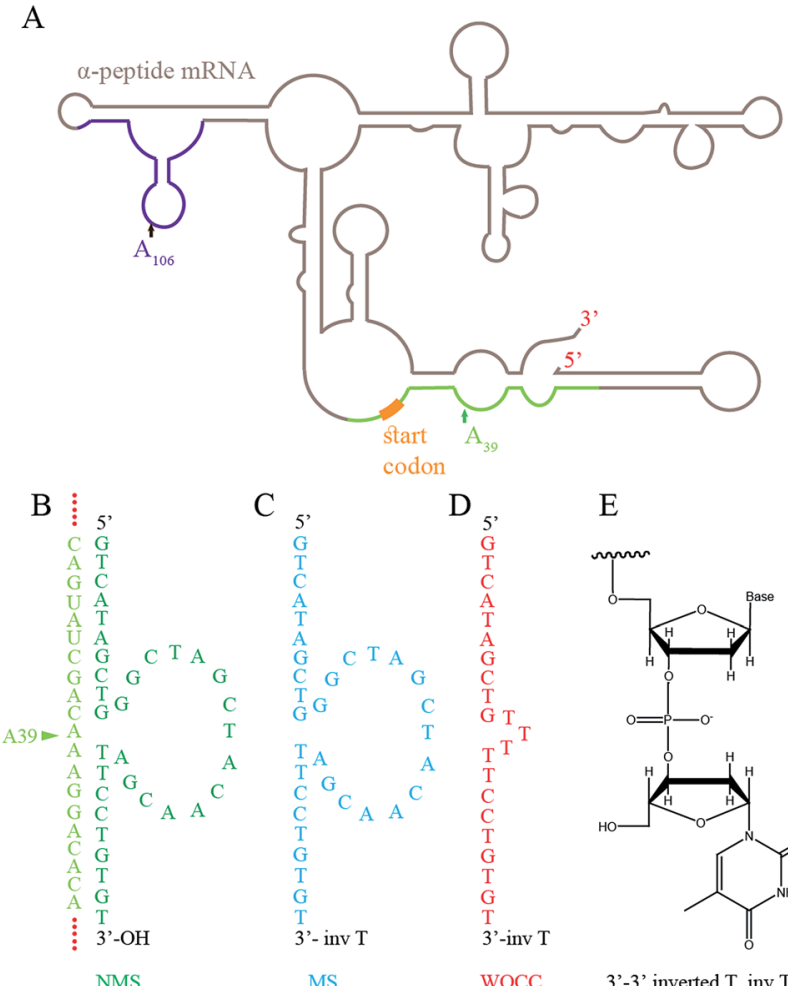

$\mathrm{E}$

MS

WOCC

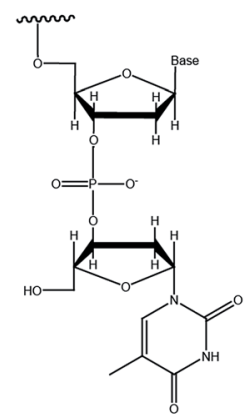

3'-3' inverted T, inv T

Fig. 1 (A) Schematic presentation of the secondary structure of the $\alpha$ peptide mRNA from pGEM-T vector. The secondary structure was predicted by mfold. Two target sites for cleavage with 10-23 deoxyribozyme were $A_{39}$, prior to the start codon ( $5^{\prime}$ UTR) and $A_{106}$ in the translated region. The sequences with which designed deoxyribozymes bind are colored light green and purple. The start codon is colored orange. (B) The nonmodified 10-23 deoxyribozyme (NMS) designed for $A_{39}$. The cleavage site is shown by an arrow. (C) The modified 10-23 (MS) deoxyribozyme has the same sequence as NMS, except with an inverted thymidine at $3^{\prime}$ end. (D) The control DNA without catalytic core (WOCC) had three thymidines instead of catalytic core and was modified with $3^{\prime}-3^{\prime}$ inverted thymidine as MS. (E) The structure of the modification used in this report.

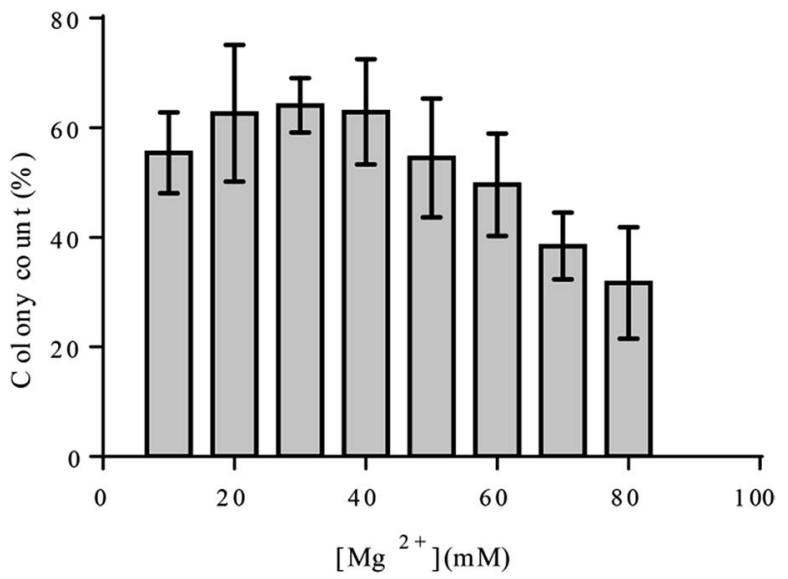

Fig. 2 Optimization of the $\mathrm{Mg}^{2+}$ concentration for formation of $E$. coli colonies.

span of $c a .12 \mathrm{~h}$. After appearance of white colonies, a solution containing both IPTG and X-gal (IPTG-X-gal) was sprayed over the agar plates. The solution was sprayed to ensure even 
distribution of the solution. The color of the colonies started to change from white to blue. The complete color change took about $12 \mathrm{~h}$ and the colonies had shown a range of blue color intensities in the meantime. ESI Fig. SI and SII $\dagger$ show exemplary agar plates prior and after IPTG-X-gal spray. The percentages of the blue color of samples with active deoxyribozymes were clearly less than control samples at early time points (Fig. SIII $\dagger$ ). The photographs and analyzed data for the case of the 10-23 deoxyribozyme for untranslated region, $A_{39}$, are shown in Fig. 3. The best effect was observed $1.5 \mathrm{~h}$ after the spray. The difference faded at later time points in a way that at $12 \mathrm{~h}$ after spray, almost no difference of the blue color was detectable in colonies. Bacterial colonies with different ranges of blue-color were grouped and plotted in Fig. 3B. Such presentation shows that the number of colonies with higher cyan percentage are reduced in MS and NMS in comparison to negative control.

In the case of the assays for untranslated region, the in negative control (NC), which contained only pGEM-T blue color started to appear rapidly and the number of colonies with high cyan percentage shifted to about $60 \%$ of the population within $1.5 \mathrm{~h}$. Such rapid color change was in favor of successful transcription of the mRNA of the $\alpha$-peptide, fruitful translation of the mRNA and active complementation of the $\beta$-galactosidase. Formation of the blue color in samples with WOCC had no significant change in comparison to the negative control and $c a$. $70 \%$ of the colony population had a cyan color between $14-27 \%$
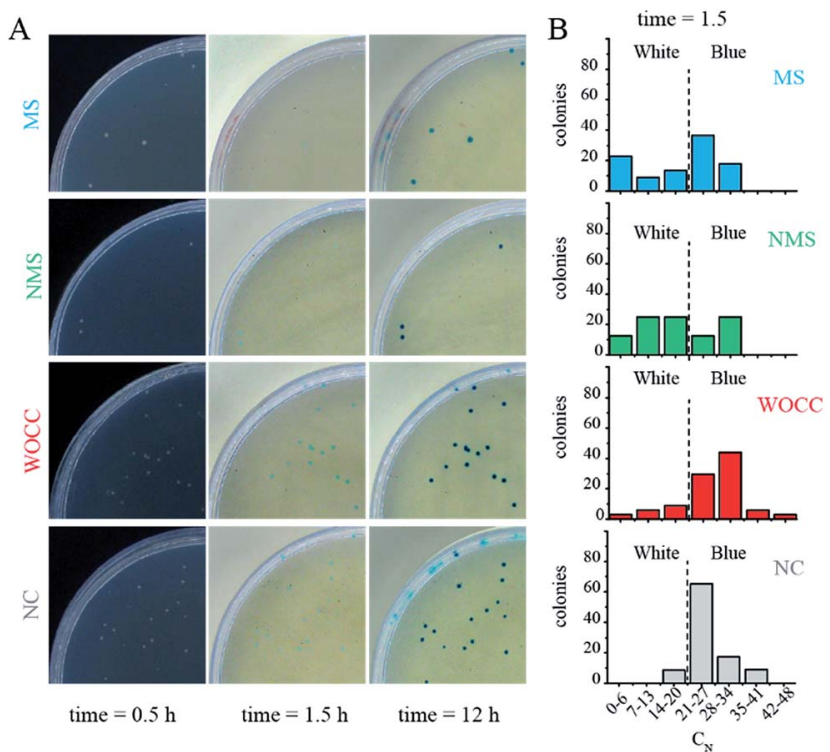

Fig. 3 (A) Photos of the agar plates 0.5, 1.5 and $12 \mathrm{~h}$ after IPTG-X-gal spray. The background at time point 0.5 is black for better illustration of the white colonies. The blue color percentage of some colonies are marked in an exemplary photo of an agar plate in Fig. SI. $\uparrow$ (B) Histogram of populations with various blue percentages at $1.5 \mathrm{~h}$ after spraying IPTG-X-gal solution for samples with deoxyribozymes against the untranslated region. The dashed lines indicate the threshold for transition to blue color colony. NC: negative control, WOCC: without catalytic core, NMS: non-modified sequence, MS: modified sequence with $3^{\prime}-3^{\prime}$ inverted thymidine. Full plate images are shown in ESI Fig. SIV.† after $1.5 \mathrm{~h}$. The color change in the other two samples, i.e. NMS and MS were stalled at early time points and the effect was more pronounced for MS. In a way that $c a .20 \%$ of the population of the colonies had minimal cyan color (0-6\%) after $1.5 \mathrm{~h}$ (Fig. 3A, second column). All the bacterial colonies could transform to complete blue color at longer time points, i.e. $12 \mathrm{~h}$ after spray (Fig. 3A, last column).

Fig. 4 depicts the average of the percentage of the cyan color in the colonies of agar plates at three-time points. For both cases of assays, with 10-23 deoxyribozymes for untranslated region, $A_{30}$ and translated region, $A_{106}$, the most difference between the experimental setups was observed at $1.5 \mathrm{~h}$ after spraying IPTG-X-gal. At this time point significant reduction of the blue color was observed with modified sequences. Presence of the $3^{\prime}$-inverted thymidine at the $3^{\prime}$ end of the deoxyribozymes was turned out to improve the catalytic performance of both deoxyribozymes that inside bacterial cells. The non-modified sequence could only reduce the cyan color in the translated region. In both cases, the WOCC DNA had no meaningful effect in comparison to the control experiments (Fig. 4).

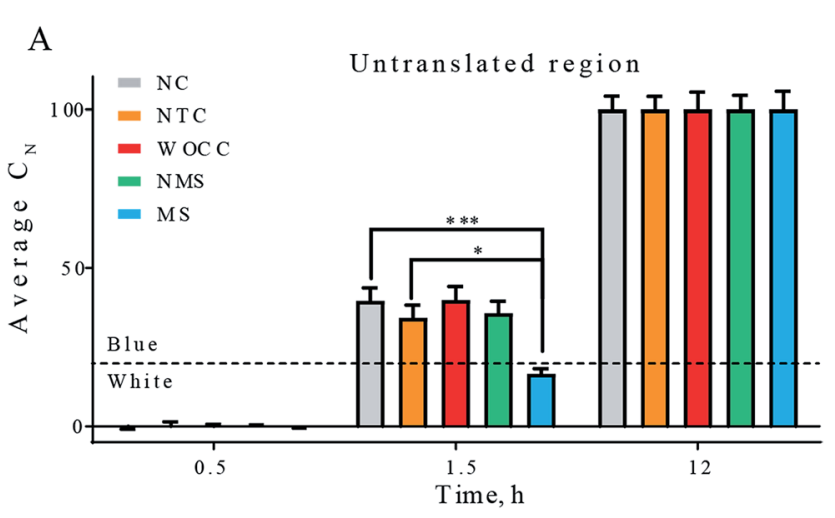

B

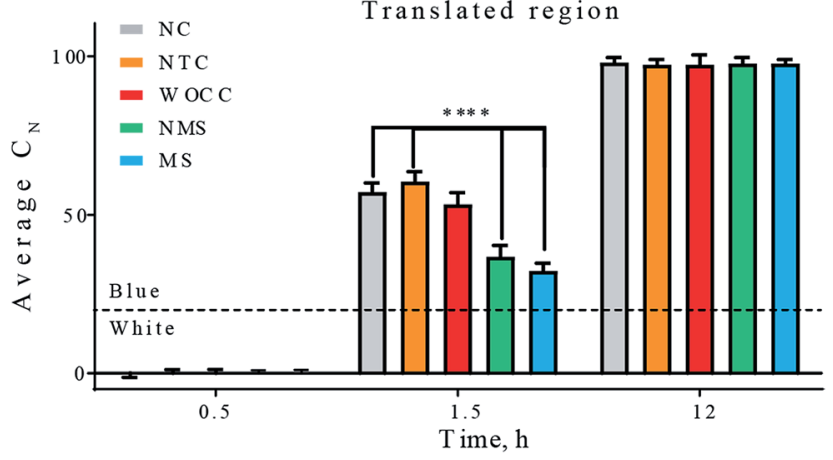

Fig. 4 Average percentage of cyan color in total number of colonies. The first column is negative control (NC) in which pGEM-T vector was transformed in the absence of additional oligonucleotide. The next four columns are the result of co-transformation of pGEM-T vector and according oligonucleotides. Non-target DNA control (NTC), WOCC, NMS and MS sequences are as in Table 2. Panel (A) untranslated region as target, panels (B) translated region as target. The indicated times are the time points after solution spray. The dashed line depicts the threshold for transition to blue color. All values reported are mean \pm SEM. [* for $p<0.05, * *$ for $p<0.01, * * *$ for $p<0.005$ and $* * * *$ for $p<0.001$. 


\section{Miller assay}

The enzyme $\beta$-galactosidase is able to hydrolyze a wide range of artificial $\beta$-D-galactopyranosides including the chromogenic substrate ortho-nitrophenyl- $\beta$-D-galactopyranoside (ONPG). The hydrolysis of the glycosidic bond results in formation of orthonitrophenol which is yellow. A method that measures the specific activities of the $\beta$-galactosidase has been presented by Miller in 1972. The assay reports the enzymatic activity as miller units.

The $\beta$-galactosidase activity of the transformed bacterial colonies were analyzed by Miller assay in this study. The yellow color was started to appear $c a .17 \mathrm{~h}$ after transformation. Since then, the Miller assays were performed for negative control, non-target control DNA, WOCC, NMS and MS. As in blue-white screening, the significant differences among the experimental setups were observed transiently. The most difference was at the $19 \mathrm{~h}$ time point. Consistent with blue-white screening results, at this time point, modified sequences of deoxyribozymes had significant reduction effect in comparison to both negative control and non-target control in both target sites. Nonmodified sequence of 10-23 was effective for reduction of the blue color in translated region, but no meaningful reduction was observed with non-modified sequence in the untranslated region, consistent with blue-white screening results. WOCC could not show significant difference in either of the cases (Fig. 5).

\section{Discussion}

The RNA-cleaving 10-23 deoxyribozyme has been widely used for gene silencing in eukaryotic cells. ${ }^{27}$ This deoxyribozyme was extensively applied for cleavage of mRNAs that are responsible for several diseases such as allergic reactions, inflammation and cancer. ${ }^{28}$ A few 10-23 deoxyribozymes have been gone through clinical trials and currently passed phase I and II. In addition, many viral mRNAs have been targeted for cleavage with 10-23 deoxyribozymes inside eukaryotic cells. ${ }^{5}$ Despite such advances in application of 10-23 deoxyribozymes in eukaryotic cells, there are only few reports on the activity of this deoxyribozyme inside prokaryotic cells (Table 1). In addition, the available reports only target bacterial growth and survival. The $\beta$-lactamase pathway was the main target for 10-23 deoxyribozymes mediated cleavage in these reports.

To the best of our knowledge, the current report is the first one for cleavage of an mRNA of a reporter gene with 10-23 deoxyribozymes in a viable prokaryotic cell. The target mRNA in this study was not intrinsically present in the host genome and was transformed simultaneously with the deoxyribozyme's oligonucleotide or its controls. An equivalent study for eukaryotic cells has been reported by Ackermann et al. for human embryonic kidney, HEK293 cell lines. ${ }^{29}$ The target gene was provided with a eukaryotic expression vector. The transformation of the expression vector and deoxyribozyme's oligonucleotide was carried out simultaneously. The ratio of vector to oligonucleotide was $8500: 1$. Although the strength of the promoters and the cell division rates of the two studies are not directly comparable, this ratio was taken as a rule of thumb for this study.
A

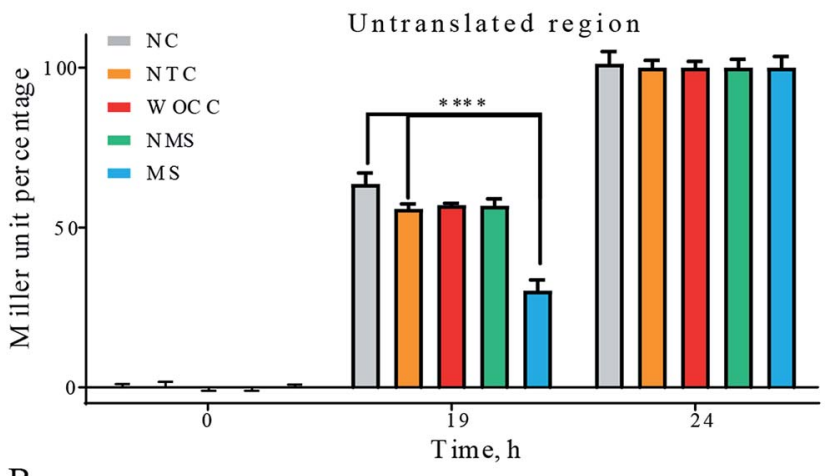

B

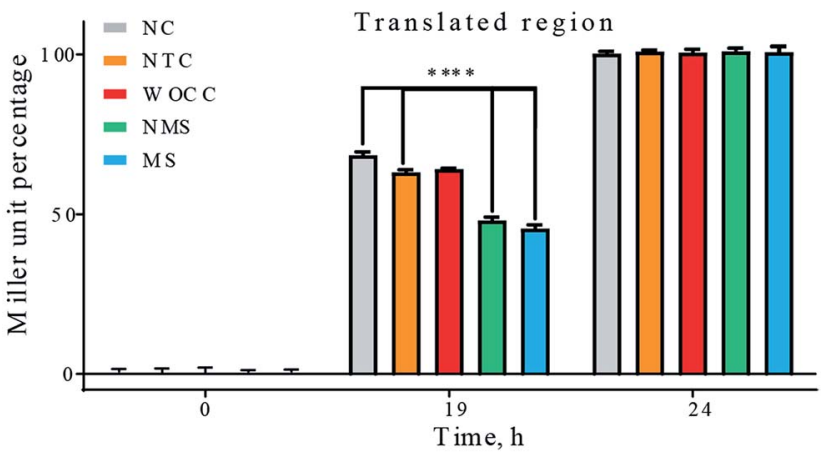

Fig. 5 Miller assay for activity of 10-23 deoxyribozyme and controls for untranslated (A) and translated (B) region. NC: negative control, NTC: non-target DNA control WOCC: without catalytic core, NMS: non-modified sequence, MS: modified sequence with $3^{\prime}-3^{\prime}$ inverted thymidine. [**** for $p<0.001$ ].

In our study, we applied 0.02 pmol vector and $600 \mathrm{pmol}$ deoxyribozyme for co-transformation into $50 \mu \mathrm{l}$ of competent cells with total number of ca. 375000000 cells. The ratio of vector to oligonucleotide was $c a .32000: 1$, and the ratio of deoxyribozyme to bacterial cells was $1000000: 1$. Despite excessive application of deoxyribozymes in this study, the transformed RNA-cleaving 10-23 deoxyribozyme was in a big challenge inside rapidly growing $E$. coli colonies. In other words, besides nuclease-mediated degradation of the transformed deoxyribozymes, 10-23 oligonucleotides were prone to dilute and vanish in bacterial descendants. Hence, we expected a transient knock-down effect which was confirmed by our results.

The blockade of formation of blue-color from X-gal was transient. In other words, the colonies with active enzyme were as blueish as control colonies at long time span (above $4 \mathrm{~h}$ from induction). The reason could be due to two parallel phenomena; (1) dilution of the deoxyribozymes upon bacterial division, (2) nuclease degradation. In this study, the deoxyribozymes had no antibiotic-like effect; therefore, the colonies were growing during the assays. This means, while the number of transformed DNA oligonucleotides was constant, the number of cells was increasing. Thus, it would be logical to deduce that within a colony there would be some bacterial cells that get a low concentration of the deoxyribozyme. At such low concentrations, the target mRNA ( $\alpha$-peptide) was not efficiently cleaved 
Table 1 Reports on application of 10-23 deoxyribozyme in prokaryotic cells

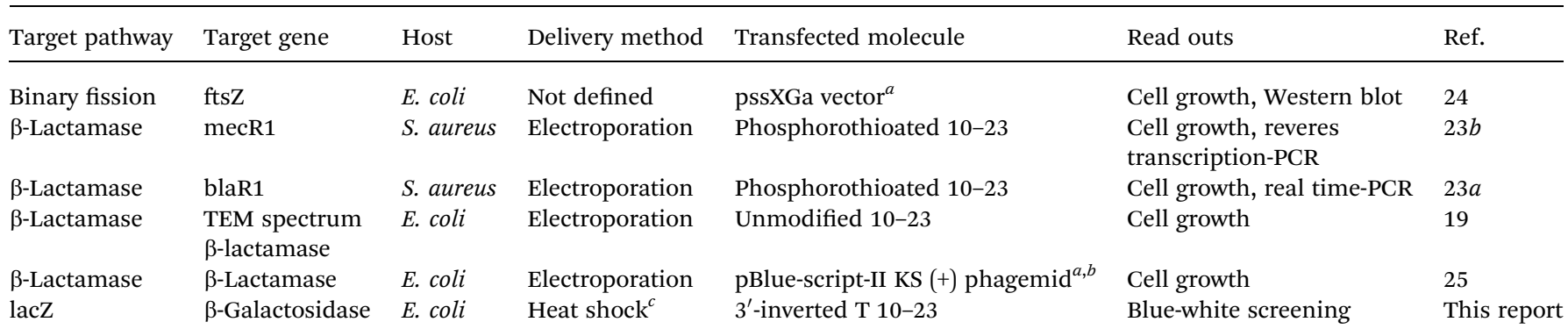

${ }^{a}$ Carrying 10-23 deoxyribozyme sequence. ${ }^{b}$ Plus helper phage VCSM13 to acquire single strand recombinant vector. ${ }^{c}$ Standard heat-shock transformation of chemically competent cells.

and cells scape from blockade of $\alpha$-complementation. If only a few cells in a colony form the active $\beta$-galactosidase the presence of blue color will override the color of the colony i.e. the colony becomes blue, in spite of probable presence of bacterial cells that lack active $\beta$-galactosidase.

A thorough study must have been performed to find the best time point with maximal difference with control samples. With 30 min intervals, we comprehensively investigated for the best time to visualize the effect. The optimal time for blue-white screening was between 1-2 $\mathrm{h}$ after induction by spraying IPTG-X-gal solution. This time was indeed 11-12 hours after transformation, due to the fact that it took about $10 \mathrm{~h}$ for formation of detectable colonies. The best time point for observing the difference in the Miller assay was $19 \mathrm{~h}$, since it took about $17 \mathrm{~h}$ for initial observation of the yellow color from Miller assay.

Regarding the nuclease dependent degradation of oligonucleotides, various modifications have been applied for 10-23 deoxyribozyme to increase the half-life of the DNA within eukaryotic cells. In contrast, the only modification in prokaryotic system was phosphorothioation. Here, we investigated the effect of the $3^{\prime}-3^{\prime}$ inverted thymidine. Our result demonstrated that MS (the 10-23 deoxyribozyme with modification) could knock-down $\beta$-galactosidase more efficiently in comparison to NMS (non-modified sequence). Formation of the blue color from X-gal and formation of yellow color from ONPG was meaningfully reduced in MS, although the effect was transient. NMS was effective only in translated region, while the MS could stall blue color formation in both target sites. Thus, we conclude that $3^{\prime}-3^{\prime}$ inverted thymidine is an efficient modification for application of deoxyribozymes in prokaryotic cells.

The DNA oligonucleotides at which the catalytic cores were replaced by three thymidines could not cause a decrease in the readout of any of the assays, expectedly. These oligonucleotides were modified with $3^{\prime}-3^{\prime}$ inverted thymidine and thus the absence of the effect was not the result of nuclease degradation. Hence, the blue- or yellow-color reductions were deduced to be the direct effect of 10-23 deoxyribozyme catalysis and cleavage of the mRNA of the $\alpha$-peptide. In the absence of $\alpha$-peptide, the four monomers of $\beta$-galactosidase could not form the active tetramer and the enzyme was blocked. Our result showed that similar reduction extent for translated and untranslated regions when modified deoxyribozymes were used. However, with nonmodified sequences, the 10-23 deoxyribozyme could not efficiently cleave and block translation of $\alpha$-peptide in untranslated region.

\section{Experimental}

\section{Materials and methods}

Chemicals and reagents. All nucleotide sequences including unmodified DNA oligonucleotides and modified DNA with $3^{\prime}-3^{\prime}$ inverted thymidine were purchased from Bioneer, Korea. Ampicillin, $\mathrm{CaCl}_{2}, \mathrm{MgCl}_{2}, \mathrm{Na}_{2} \mathrm{HPO}_{4}, \mathrm{KCl}, \mathrm{MgSO}_{4}$, mercaptoethanol, chloroform and sodium dodecyl sulfate (SDS), sodium carbonate, isopropyl $\beta$-D-1-thiogalactopyranoside (IPTG), $O$ nitrophenyl-D-galactopyranoside (ONPG) and 5-bromo-4-chloro3 -indolyl- $\beta$-D-galactopyranoside (X-gal) were purchased from Sigma-Aldrich.

Selection of host and vector. In this report, PGEM-T was selected as a model vector for the $\alpha$-complementation. The $E$. coli DH5 $\alpha$ was chosen as the host strain since it has a deletion at amino-terminus (lacZDM15) and is suitable for blue-white screening. pGEM-T vector plasmid was purchased from SinaClone, Tehran, Iran. DH5 $\alpha$ strain with ampicillin resistance was purchased from Persian type culture collection, Tehran, Iran.

Design of deoxyribozymes. The sequence of pGEM-T vector was retrieved from addgene web page. The transcript of $\alpha$ peptide fragment was recouped as the reverse complementary sequence after the promoter, since the promoter was in reverse orientation. The sequence of the open reading frames was compared with the original sequence of the $\alpha$-peptide with ExPASy and identity of the sequences were confirmed.

The secondary structure of the mRNA transcript of the $\alpha$ peptide was predicted with mfold from the DINAmelt web server. ${ }^{30}$ The default parameters were used, and the secondary structure with the lowest free energy was exploited to identify the regions within the mRNA that are accessible to the deoxyribozyme. The deoxyribozymes with 9 nucleotides long binding arms at each site have been designed against target sequences at $A_{39}-C_{40}$ and $A_{106}-C_{107}$. The left binding arms fully hybridize to the target DNA sequence except at the cleavage site's adenosines. The catalytic core of the 10-23 deoxyribozyme with the sequence of GGCTAGCTACAACGA were substituted the base 
pair at this adenosine. The adjacent cytosines at the cleavage sites and its $3^{\prime}$ nucleotides were hybridized to the right binding arms. The designed binding arms were blasted to assure absence of non-target binding of the deoxyribozymes. The designed deoxyribozyme sequences were either modified (here mentioned as MS = modified sequence) by adding a reversed $3^{\prime}$ thymidine or not modified (NMS).

An oligonucleotide sequence from designed deoxyribozyme without catalytic core (WOCC) was considered as a control. The catalytic domain of 10-23 deoxyribozyme was substituted with three consecutive thymidines in WOCC oligonucleotides. Additionally, non-enzymatic, and non-target control DNA (NTC) which was a random oligonucleotide sequences was used to control the effect of transformation. Table 2 depicts the oligonucleotide sequences that have been used in this study.

Transformation of the chemically competent cells. For transformation, chemically competent cells were freshly prepared starting from $1.5 \mathrm{ml}$ bacterial cells with $\mathrm{OD}_{600}$. The final volume of competent cells was $100 \mu \mathrm{l}$ from which $50 \mu \mathrm{l}$ was used for transformation. Single stranded oligonucleotides were co-transformed with p-GEM plasmids for $\alpha$-complementation simultaneously. Calcium chloride treated competent cells were incubated in presence of $18 \mathrm{ng}$ i.e. $0.02 \mathrm{pmol}$ PGEM-T vector and $600 \mathrm{pmol}$ of oligonucleotides on ice for $30 \mathrm{~min}$. Heat shock were performed by incubation at $42{ }^{\circ} \mathrm{C}$ in a water bath for $90 \mathrm{~s}$ followed by 2 min incubation on ice. Subsequently, $400 \mu \mathrm{l}$ of LB medium without antibiotic were added to the heat-shocked mixtures and incubated at $37^{\circ} \mathrm{C}$ for $1 \mathrm{~h}$. Samples were centrifuged at $8000 \mathrm{rpm}$, room temperature and the pallets were resuspended in $50 \mu \mathrm{l}$ of LB medium. The re-suspended bacteria were sub-cultured on agar plates containing ampicillin and optimized concentration of magnesium for blue-white screening assays and were sub-cultured in LB medium with ampicillin for miller assays.

Blue-white screening. After transformation, plating cells on agar and appearance of the first colonies (12 h), $150 \mu \mathrm{l}$ of a solution containing $1.2 \%$ IPTG (for induction of the expression of the $\beta$-galactosidase) and $0.5 \% \mathrm{X}$-gal (chromogenic lactose analogue) sprayed on the agar plates. The white colonies started to synthesize $\beta$-galactosidase and produce blue color. The agar plates were placed into a photo chamber with a yellow or black paper beneath. The yellow color was chosen since it is the most color to differentiate between white and blue color.
The chamber was equipped with white light LED strips, spanning all four vertical walls of the chamber. A camera was placed on top of the chamber and photographs were taken at fixed intervals (every $30 \mathrm{~min}$ ). Photos were analyzed with Adobe Photoshop CC2014 by reporting the percentage of the cyan in the Cyan-Magenta-Yellow-Black (CMYK) color system. In the CMYK system, each of the colors have a specific value between 0-100 that better defines the blue color of a figure in comparison to Red-Green-Blue (RGB) system. The cyan percentage of the white colonies in an un-induced plate was between $40-50 \%$ (Fig. SI $\dagger$ ). Any increase in the cyan percentage was deduced to be upon activity of $\beta$-galactosidase; accordingly, the minimal cyan percentage of such plate was taken as the $C_{\text {background }}$ and was deducted from all cyan percentages of colonies of plates that got induced with IPTG-X-gal. The residual cyan percentages were normalized in a scale of 1-100. White color colonies have $C_{\mathrm{N}}$ values below 20 . Normalized $C$ values, $C_{\mathrm{N}}$, were plotted by Graphpad Prism 7. All experiments were performed three times. The graphs show mean \pm standard error of means (SEM).

Miller assay. Miller assay was performed according to standard protocols. ${ }^{31}$ Briefly, the transformed bacterial cells were transferred to $1.5 \mathrm{ml} \mathrm{LB}$ media with ampicillin and magnesium chloride. Next, $5 \mu$ IPTG $20 \%$ was added to induce expression of $\beta$-galactosidase. At specific time points, $20 \mu \mathrm{l}$ of the medium were mixed with $80 \mu \mathrm{l}$ of the freshly made permeating buffer (0.1 $\mathrm{M} \mathrm{Na}_{2} \mathrm{HPO}_{4}, 20 \mathrm{mM} \mathrm{KCl,} 2 \mathrm{mM} \mathrm{MgSO}$, 0.0001\% v/v mercaptoethanol). Then $2.5 \mu \mathrm{l}$ chloroform and $2.5 \mu \mathrm{l} 20 \%$ SDS were directly added to the mixture. Samples were vortexed-spinned and incubated for $20 \mathrm{~min}$ at $30{ }^{\circ} \mathrm{C}$. Consequently, $600 \mu \mathrm{l} 1 \%$ ONPG was added. The ONPG substrate was supplemented with fresh mercaptoethanol (2.7 $\mu \mathrm{l}$ for each $1 \mathrm{ml}$ of the solution) before to addition. Expressed $\beta$-galactosidases cleave ONPG to lactose and $O$-nitrophenol. The latter have a yellow color and absorbs at $420 \mathrm{~nm}$. $\beta$-Galactosidase reaction was stopped by addition of $700 \mu \mathrm{l} 10 \% \mathrm{Na}_{2} \mathrm{CO}_{3}$, raising the $\mathrm{pH}$ of the solution to 9.0. Specific activity of the $\beta$-galactosidase was measured in Miller scale. Miller unit was defined as in eqn (1). Where, $A_{420}$ is absorption of yellow color produced in the test and $A_{550}$ is scattered from cellular debris that was corrected by the factor of 1.75 and $A_{600}$ determined cell density demonstrator.

Miller unit $=1000 \times\left[\left(A_{420}-1.75 \times A_{550}\right) /\right.$ $\left(A_{600} \times\right.$ volume $\times$ reaction time $\left.)\right]$

Table 2 Design of 10-23 deoxyribozymes and control oligonucleotides ${ }^{a}$

\begin{tabular}{|c|c|c|}
\hline Target & Oligonucleotide & Sequence \\
\hline \multirow[t]{2}{*}{ Untranslated region $A_{39}-C_{40}$} & NMS & GTCATAGCTG GGCTAGCTACAACGA TTCCTGTGT \\
\hline & MS & GTCATAGCTG $\overline{\text { GGCTAGCTACAACGA }}$ TTCCTGTGT-inv T \\
\hline \multirow[t]{3}{*}{ Translated region $A_{106}-C_{107}$} & NMS & TCCCAACGCG GGCTAGCTACAACGA TGGATGCAT \\
\hline & MS & TCCCAACGCG GGCTAGCTACAACGA TGGATGCAT-inv T \\
\hline & WOCC & TCCCAACGCG $\overline{\text { TTT TGGATGCAT-inv T }}$ \\
\hline
\end{tabular}

${ }^{a}$ NMS: non-modified 10-23 deoxyribozyme sequence, MS: modified 10-23 deoxyribozyme sequence with $3^{\prime}-3^{\prime}$ inverted thymidine, WOCC: without catalytic core. The sequence of the catalytic core or its substitutes are bold and underlined. 
Statistical analysis. All values of analyzed data are presented as mean \pm standard deviation (SD) or standard error (SE) from three replications. The analysis method was one-way ANOVA followed by multiple comparison test (GraphPad Prism software version 7). Differences among the groups were considered statistically significant when the $p$ value was less than 0.05 or indicated values in the graphs.

\section{Conclusions}

In summary, this report showed a proof of concept for application of blue-white screening as a readout system for activity of the model 10-23 deoxyribozyme. The result of this report can promote future investigations on activity of RNA-cleaving deoxyribozymes in vivo. The applied condition provided a transient inhibition effect on $\beta$-galactosidase activity. Stable knockdown effect is expected by employing methods that provide in situ production of single stranded deoxyribozymes in prokaryotic cells e.g. pBlue-scriptIIKS $(+)^{25}$ or MMLV-RT system. ${ }^{7}$

\section{Conflicts of interest}

None of the authors declare conflict of interest.

\section{Acknowledgements}

The authors would very much like to thank University of Isfahan for its support of this study.

\section{Notes and references}

1 T. R. Cech, The Ribosome Is a Ribozyme, Science, 2000, 289(5481), 878-879.

2 (a) P. Chartrand, S. C. Harvey, G. Ferbeyre, N. Usman and R. Cedergren, An oligodeoxyribonucleotide that supports catalytic activity in the hammerhead ribozyme domain, Nucleic Acids Res., 1995, 23(20), 4092-4096; (b) R. R. Breaker and G. F. Joyce, A DNA enzyme that cleaves RNA, Chem. Biol., 1994, 1(4), 223-229.

3 Y. Lee, P. C. Klauser, B. M. Brandsen, C. Zhou, X. Li and S. K. Silverman, DNA-Catalyzed DNA Cleavage by a Radical Pathway with Well-Defined Products, J. Am. Chem. Soc., 2017, 139(1), 255-261.

4 S. W. Santoro and G. F. Joyce, A general purpose RNAcleaving DNA enzyme, Proc. Natl. Acad. Sci. U. S. A., 1997, 94(9), 4262-4266.

5 N. Singh, A. Ranjan, S. Sur, R. Chandra and V. Tandon, Inhibition of HIV-1 Integrase gene expression by 10-23 DNAzyme, J. Biosci., 2012, 37(3), 493-502.

6 A. A. Fokina, D. A. Stetsenko and J. C. Francois, DNA enzymes as potential therapeutics: towards clinical application of 1023 DNAzymes, Expert Opin. Biol. Ther., 2015, 15(5), 689-711.

7 J. Li, N. Wang, Q. Luo and L. Wan, The 10-23 DNA enzyme generated by a novel expression vector mediate inhibition of taco expression in macrophage, Oligonucleotides, 2010, 20(2), 61-68.
8 L. Robaldo, A. Berzal-Herranz, J. M. Montserrat and A. M. Iribarren, Activity of core-modified 10-23 DNAzymes against HCV, ChemMedChem, 2014, 9(9), 2172-2177.

9 K. Yehl, J. P. Joshi, B. L. Greene, R. B. Dyer, R. Nahta and K. Salaita, Catalytic Deoxyribozyme-Modified Nanoparticles for RNAi-Independent Gene Regulation, ACS Nano, 2012, 6(10), 9150-9157.

$10 \mathrm{H}$. Unwalla and A. C. Banerjea, Novel mono- and di-DNAenzymes targeted to cleave TAT or TAT-REV RNA inhibit HIV-1 gene expression, Antiviral Res., 2001, 51(2), 127-139.

11 S. Kim, S.-R. Ryoo, H.-K. Na, Y.-K. Kim, B.-S. Choi, Y. Lee, D.-E. Kim and D.-H. Min, Deoxyribozyme-loaded nanographene oxide for simultaneous sensing and silencing of the hepatitis $\mathrm{C}$ virus gene in liver cells, Chem. Commun., 2013, 49(74), 8241-8243.

12 B. Kumar, P. Kumar, R. Rajput, L. Saxena, M. K. Daga and M. Khanna, Sequence-specific cleavage of BM2 gene transcript of influenza B virus by 10-23 catalytic motif containing DNA enzymes significantly inhibits viral RNA translation and replication, Nucleic Acid Ther., 2013, 23(5), 355-362.

13 P. Reyes-Gutierrez and L. M. Alvarez-Salas, Cleavage of HPV16 E6/E7 mRNA mediated by modified 10-23 deoxyribozymes, Oligonucleotides, 2009, 19(3), 233-242.

$14 \mathrm{~S}$. Wu, J. Xu, J. Liu, X. Yan, X. Zhu, G. Xiao, L. Sun and P. Tien, An efficient RNA-cleaving DNA enzyme can specifically target the 5 '-untranslated region of severe acute respiratory syndrome associated coronavirus (SARS-CoV), $J$. Gene Med., 2007, 9(12), 1080-1086.

15 Y. Cao, L. Yang, W. Jiang, X. Wang, W. Liao, G. Tan, Y. Liao, Y. Qiu, D. Feng and F. Tang, Therapeutic evaluation of Epstein-Barr virus-encoded latent membrane protein-1 targeted DNAzyme for treating of nasopharyngeal carcinomas, Mol. Ther., 2014, 22(2), 371-377.

16 A.-Y. Xing, D.-B. Shi, W. Liu, X. Chen, Y.-L. Sun, X. Wang, J.-P. Zhang and P. Gao, Restoration of chemosensitivity in cancer cells with MDR phenotype by deoxyribozyme, compared with ribozyme, Exp. Mol. Pathol., 2013, 94(3), 481-485.

17 U. Homburg, H. Renz, W. Timmer, J. M. Hohlfeld, F. Seitz, K. Lüer, A. Mayer, A. Wacker, O. Schmidt, J. Kuhlmann, A. Turowska, J. Roller, K. Kutz, G. Schlüter, N. Krug and H. Garn, Safety and tolerability of a novel inhaled GATA3 mRNA targeting DNAzyme in patients with TH2-driven asthma, J. Allergy Clin. Immunol., 2015, 136(3), 797-800.

18 N. Krug, J. M. Hohlfeld, A.-M. Kirsten, O. Kornmann, K. M. Beeh, D. Kappeler, S. Korn, S. Ignatenko, W. Timmer, C. Rogon, J. Zeitvogel, N. Zhang, J. Bille, U. Homburg, A. Turowska, C. Bachert, T. Werfel, R. Buhl, J. Renz, H. Garn and H. Renz, Allergen-Induced Asthmatic Responses Modified by a GATA3-Specific DNAzyme, $N$. Engl. J. Med., 2015, 372(21), 1987-1995.

19 F. Chen, Z. Li, R. Wang, B. Liu, Z. Zeng, H. Zhang and J. Zhang, Inhibition of ampicillin-resistant bacteria by novel mono-DNAzymes and di-DNAzyme targeted to betalactamase mRNA, Oligonucleotides, 2004, 14(2), 80-89. 
20 A. Abdelgany, M. K. Uddin, M. Wood, K. Taira and D. Beeson, Design of efficient DNAzymes against muscle AChR alpha-subunit cRNA in vitro and in HEK 293 cells, $J$. RNAi Gene Silencing, 2005, 1(2), 88-96.

21 M. Chakravarthy, M. T. Aung-Htut, B. T. Le and R. N. Veedu, Novel Chemically-modified DNAzyme targeting Integrin alpha-4 RNA transcript as a potential molecule to reduce inflammation in multiple sclerosis, Sci. Rep., 2017, 7, 1613.

22 A. A. Fokina, M. I. Meschaninova, T. Durfort, A. G. Venyaminova and J. C. Francois, Targeting insulinlike growth factor I with 10-23 DNAzymes: 2'-O-methyl modifications in the catalytic core enhance mRNA cleavage, Biochemistry, 2012, 51(11), 2181-2191.

23 (a) Z. Hou, J. R. Meng, J. R. Zhao, B. Q. Hu, J. Liu, X. J. Yan, M. Jia and X. X. Luo, Inhibition of beta-lactamase-mediated oxacillin resistance in Staphylococcus aureus by a deoxyribozyme, Acta Pharmacol. Sin., 2007, 28(11), 17751782; (b) Z. Hou, J. R. Meng, C. Niu, H. F. Wang, J. Liu, B. Q. Hu, M. Jia and X. X. Luo, Restoration of antibiotic susceptibility in methicillin-resistant Staphylococcus aureus by targeting mecR1 with a phosphorothioate deoxyribozyme, Clin. Exp. Pharmacol. Physiol., 2007, 34(11), 1160-1164.

24 X. X. Tan, K. Rose, W. Margolin and Y. Chen, DNA enzyme generated by a novel single-stranded DNA expression vector inhibits expression of the essential bacterial cell division gene ftsZ, Biochemistry, 2004, 43(4), 1111-1117.
25 Y. Sheng, Z. Zeng, W. Peng, D. Jiang, S. Li, Y. Sun and J. Zhang, Design and switch of catalytic activity with the DNAzyme-RNAzyme combination, FEBS Lett., 2007, 581(9), 1763-1768.

26 D. H. Juers, B. W. Matthews and R. E. Huber, LacZ $\beta$ galactosidase: Structure and function of an enzyme of historical and molecular biological importance, Protein Sci., 2012, 21(12), 1792-1807.

27 C. R. Dass, P. F. Choong and L. M. Khachigian, DNAzyme technology and cancer therapy: cleave and let die, Mol. Cancer Ther., 2008, 7(2), 243-251.

28 M. Zhang, G. P. Drummen and S. Luo, Anti-insulin-like growth factor-iiP3 Dnazymes inhibit cell proliferation and induce caspase-dependent apoptosis in human hepatocarcinoma cell lines, Drug Des., Dev. Ther., 2013, 7, 1089.

29 J. M. Ackermann, S. Kanugula and A. E. Pegg, DNAzymemediated silencing of ornithine decarboxylase, Biochemistry, 2005, 44(6), 2143-2152.

30 N. R. Markham and M. Zuker, DINAMelt web server for nucleic acid melting prediction, Nucleic Acids Res., 2005, 33(Web Server issue), W577-W581.

31 T. Tschirhart, X. Y. Zhou, H. Ueda, C.-Y. Tsao, E. Kim, G. F. Payne and W. E. Bentley, Electrochemical Measurement of the $\beta$-Galactosidase Reporter from Live Cells: A Comparison to the Miller Assay, ACS Synth. Biol., 2016, 5(1), 28-35. 